\title{
On-line detection of the breakage of small diameter drills using current signature wavelet transform ${ }^{1}$
}

\author{
Li Xiaoli \\ Department of Mechanical Engineering, Harbin Institute of Technology, P.O. Box 422, Harbin 150001, P.R. China
}

Received 15 April 1997

\begin{abstract}
This paper presents on-line tool breakage detection of small diameter drills by monitoring the AC servo motor current. The continuous wavelet transform was used to decompose the spindle AC servo motor current signal and the discrete wavelet transform was used to decompose the feed AC servo motor current signal in time-frequency domain. The tool breakage features were extracted from the decomposed signals. Experimental results show that the proposed monitoring system possessed an excellent on-line capability; in addition, it had a low sensitivity to change of the cutting conditions and high success rate for the detection of the breakage of small diameter drills. (c) 1998 Elsevier Science Ltd. All rights reserved.
\end{abstract}

Keywords: Tool breakage; Wavelet transform; AC servo motor current signal; Drilling

\section{Introduction}

It is well known that on-line tool condition monitoring has a great significance in modern manufacturing processes. To prevent possible damages to the workpiece and machine tool, reliable sensing techniques are required for providing a rapid response to an unexpected tool breakage [1].

Reliable and industrially acceptable sensors are required for monitoring the drilling processes have been developed over the years. Several techniques have been developed to the detection of tool breakage, the most common techniques reported in the industrial machining environment are force, acoustic emission (AE) and current [2]. Force-measuring is a good method for detecting tool breakage. However, its main disadvantage is that each tool requires to be fitted with the

\footnotetext{
${ }^{1}$ Supported by the China Defense Foundation.
} 
sensor system, making this alternation very expensive; in addition, the installation of a forcemeasuring sensor system is difficult or impossible. In recent years, AE sensing technique is considered to be one of the most effective methods for tool breakage monitoring. One of the main obstacles in its application is how to detect the AE signals in rotating tool such as in Machine Center (MC) for boring and milling, or how and where to install the AE sensor. In addition, it is difficult to process $\mathrm{AE}$ signal and its structure is high expensive.

During the cutting processes, motor current is related to the tool conditions. Less power is consumed when using a breakage tool compared to a sharp tool, this variance can be exploited for on-line tool breakage monitoring. The motor current of $\mathrm{MC}$ was measured through a current transformer (such as Hall Current Sensor), measured signal was processed, the result was found to drop instantaneously and soon recover to a level prior to the drop, when tool breakage occurred [3]. The current measurement system is relatively simple and its mounting will not affect the machining operations [4]. It is less sensitive for small tool breakage when compared to force sensing and $\mathrm{AE}$ sensing. However, the current measurement system was found to be reliable in monitoring tool breakage at medium and heavy cuts [5].

Spectral analysis and time series method are the most commonly used signal processing techniques in tool breakage detection. But the above methods are such that it has a good solution only in the frequency domain and a poor solution in the time domain so that it lose some signal information in time domain, it is fitted to process stability stochastic signal. Recently, the wavelet transform (WT) proposed is a significant new tool in signal analysis and processing [6,7]. It has been used to analyze tool breakage monitoring signal $[8,9]$. WT has a good solution in the timefrequency domain so that it can extract more information in the time domain at different frequency bands [10].

Drilling is one of the most fundamental machining operations. There are many practical problems in drilling, one of the most crucial issues is tool breakage, in particular with diameters $<5 \mathrm{~mm}$ [11].

This paper presents a WT application for on-line detection of the breakage of small diameter drills, a.c. servo motor current signals are analyzed by WT and permit a simple signal processing algorithm to be used to determine the tool conditions from the WT of the AC current signal, in particular, after WT of the AC servo motor current signal. Experimental results show that current measurement system possessed reliability for detection of the of breakage of small diameter drills.

\section{Wavelet transform}

Wavelet transforms have become well known as useful tools for various signals processing applications. The WT is best suited to signal analysis [12]. Given a time varying signal $f(t)$; wavelet transforms consist of computing coefficient that inner products of the signal and a family of wavelets. In a continuous wavelet transform (CWT), the wavelet corresponding to scale $a$ and time location $b$ is

$$
\psi_{a, b}=\frac{1}{\sqrt{|a|}} \psi\left(\frac{t-b}{a}\right) a, b \in R, a \neq 0
$$

where $a$ and $b$ are the dilation and translation parameters, respectively. 
The continuous wevelet transform was defined as follows

$$
C W T\{x(t) ; a, b\}=\int x(t) \psi_{a, b} *(t) \mathrm{d} t
$$

where $*$ denotes the complex conjugation. In this paper Morlet wavelet function was used to deal with spindle motor current signal. Morlet wavelet is

$$
\psi(t)=\mathrm{e}^{-t^{2} / 2} \mathrm{e}^{i w_{0} t}
$$

its continue wavelet is

$$
\psi_{a, b}=\frac{1}{\sqrt{|a|}} \mathrm{e}^{-1 / 2((t-b) / a)^{2}} \mathrm{e}^{j w_{0}((t-b) / a)}
$$

In the above equation, $a$ and $w_{0}$ can be changed, each way yields a different type of wavelet transform. The sample frequency of the wavelet function, and the signal is $f_{w}$ and $f_{s}$ respectively, the relationship of the parameter $a$ and $w_{0}$ is

$$
a=\frac{w_{0} f_{s}}{2 \pi f_{w} f_{0}}
$$

where $f_{0}$ is frequency focused signal energy

When $a=2^{j}, b=k 2^{j}, j, k \in Z$, the wavelet are in this case

$$
\psi_{j, k}=2^{-j / 2} \psi\left(2^{-j} t-k\right)
$$

The discrete wavelet transform (DWT) is defined

$$
c_{j, k}=\int f(t) \psi_{j, k}^{*}(t)
$$

where $c_{j, k}$ is defined as wavelet coefficient, it may be though a time frequency map of the original signal $f(t)$.

Here, a multi-resolution analysis approach is used in which a discrete scaling function

$$
\phi_{j, k}=2^{-j / 2} \phi\left(\frac{t-2^{j} k}{2^{j}}\right)
$$

set

$$
d_{j, k}=\int f(t) \phi_{j, k}^{*}(t)
$$

where $d_{j, k}$ is called as scaling coefficients, it is the sampled version of original signal, when $j=$ 0 , it is the sampled version of the original. The DWT computes wavelet coefficients $c_{j, k}$ for $j=$ $1, \ldots, J$, and scaling coefficients $d_{j, k}$ given by

$$
c_{j, k}=\sum_{n} x[n] h_{j}\left[n-2^{j} k\right]
$$

and 


$$
d_{j, k}=\sum_{n} x[n] g_{j}\left[n-2^{j} k\right]
$$

where $x[n]$ are discrete-time signals, $h_{j}\left[n-2^{j} k\right]$ is the analysis discrete wavelets, the discrete equivalents to $2^{-j / 2} \psi\left(2^{-j}\left(t-2^{j} k\right)\right), g_{j}\left[n-2^{j} k\right]$ are called scaling sequence.

At each resolution $j>0$, the scaling coefficients and the wavelet coefficients

$$
\begin{aligned}
& c_{j+1, k}=\sum_{n} g[n-2 k] d_{j, k} \\
& d_{j+1, k}=\sum_{n} h[n-2 k] d_{j, k}
\end{aligned}
$$

From a mathematical point of view, the structure of computations in a DWT is exactly an octave-band filter band [13]. The terms $g$ and $h$ are high-pass and low-pass filters derived from the analysis wavelet $\psi(t)$ and the scaling function $\phi(t)$. Hence, $c_{j, k}$ represents the high frequency components of the signal $f(t)$. In the paper, DWT is used to analyze feed driver AC servo motor current signal, we employ the filters is

$$
h[]=[0.125,0.375,0.375,0.125], g[]=[-2.0,2.0] \text {. }
$$

\section{Experimental set-up}

The schematic diagram of the experimental set-up is shown in Fig. 1. Cutting tests were performed on a Machine Center Makino-FNC74-A20. The four axes (spindle, X, Y, and Z) of the machine have recalculating ball screw drives and are directly driven by permanent magnet synchronous AC servo motors. The AC servo motor current signals of the MC were measured

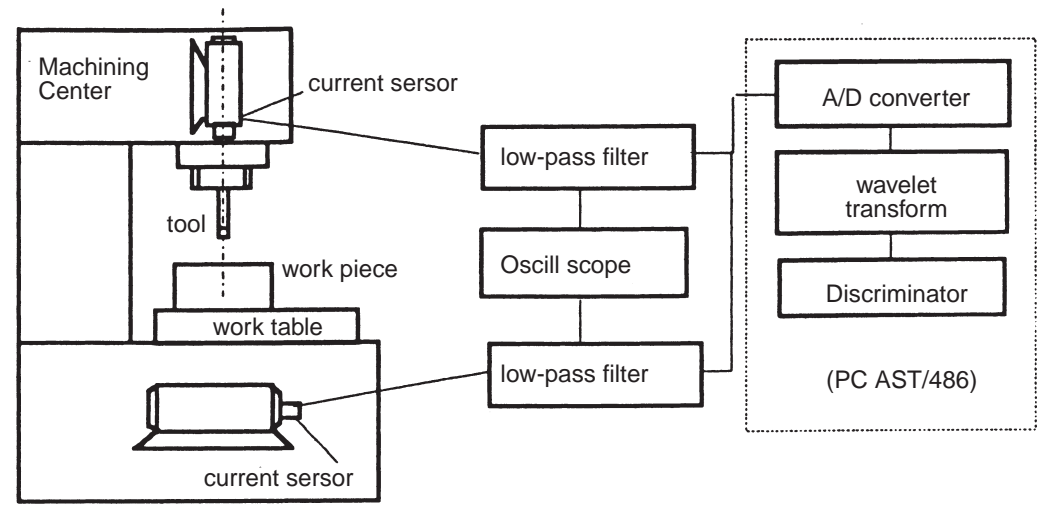

Fig. 1. Schematic diagram of the experimental set-up. 
Table 1

Experimental condition

\begin{tabular}{ll} 
Tool & HSS-drill \\
& Diameter 1, 2, 3, 4.5 mm \\
& Tool material high-speed steel \\
Cutting conditions & Spindle speed $250,300,450 \mathrm{r} / \mathrm{min}$ \\
& Feed rate $25,30,45 \mathrm{~mm} / \mathrm{min}$ \\
Workpiece & Without coolant \\
& 45\# quench steel \\
\hline
\end{tabular}
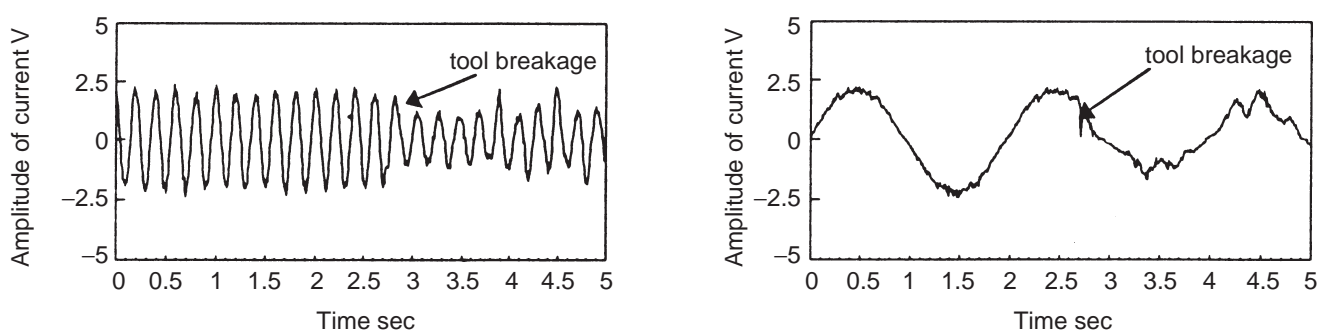

Fig. 2. Live tool breakage current signals, cutting speed: $250 \mathrm{r} / \mathrm{min}$, feed: $30 \mathrm{~mm} / \mathrm{min}$, drill diameter: $2 \mathrm{~mm}$. (a) Spindle current signal; (b) feed current signal.

through the Hall Current Sensor. The signals were firstly passed though low-pass filters (cut off frequency: $500 \mathrm{~Hz}$ ); and we sent via an A/D converter to a personal computer.

A successful tool breakage detecting method must be sensitive to tool change in tool condition, but insensitive to the variations of cutting conditions. Hence, cutting tests were conducted at different conditions to evaluate the performance of the proposed method. Table 1 shows the tool parameters and cutting conditions.

\section{WT analysis and tool breakage detection strategy}

Fig. 2(a) and (b) show the spindle current signal and feed current signal of AC servo motor, respectively. Fig. 3(a) and (b) show the result of CWT analysis of the spindle current and the
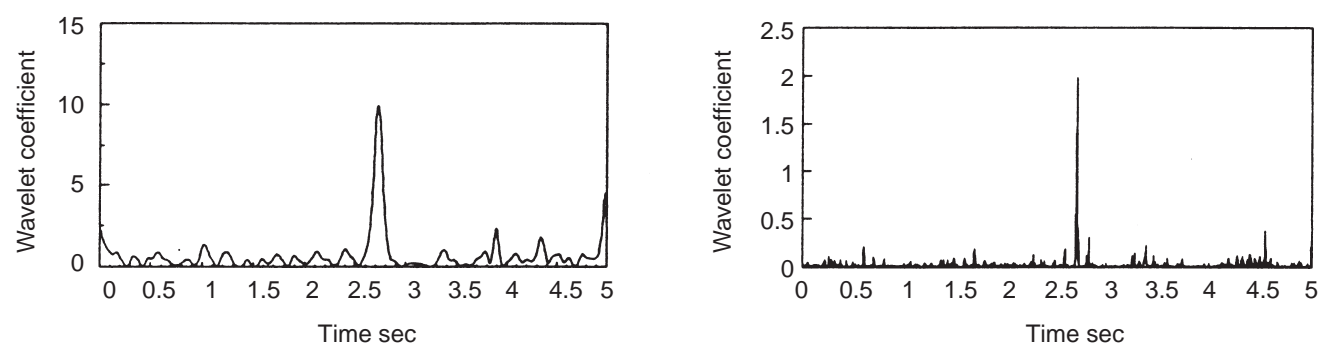

Fig. 3. WT of current signal, cutting speed: $250 \mathrm{r} / \mathrm{min}$, feed: $30 \mathrm{~mm} / \mathrm{min}$, drill diameter: $2 \mathrm{~mm}$. (a) CWT of the spindle current signal; (b) DWT of feed current signal, resolution $j=2$. 

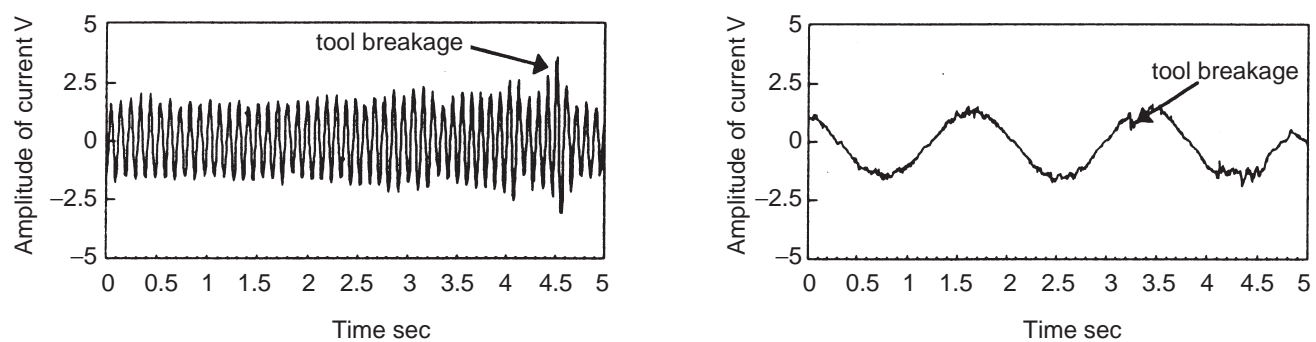

Fig. 4. Live tool breakage current signals, cutting speed: $300 \mathrm{r} / \mathrm{min}$, feed: $25 \mathrm{~mm} / \mathrm{min}$, drill diameter $3 \mathrm{~mm}$. (a) Spindle current signal; (b) feed current signal.
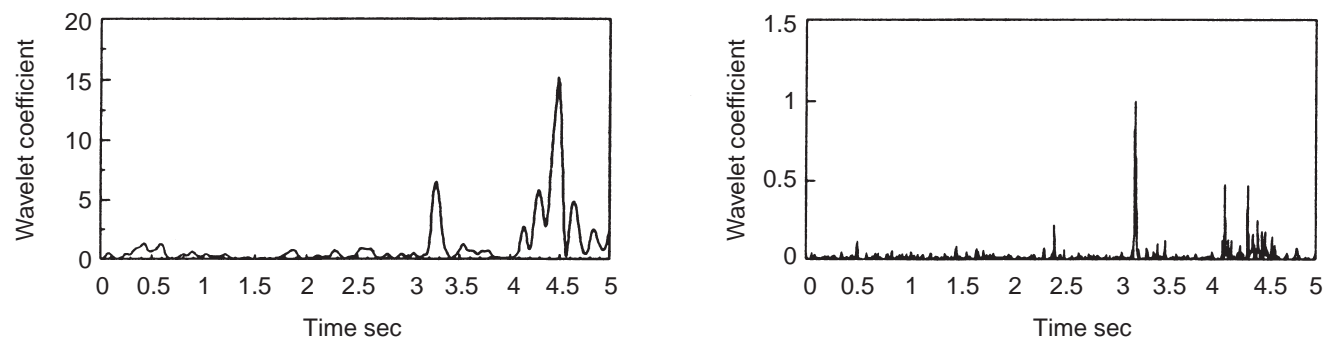

Fig. 5. WT of current signal, cutting speed: $300 \mathrm{r} / \mathrm{min}$, feed: $25 \mathrm{~mm} / \mathrm{min}$, drill diameter: $3 \mathrm{~mm}$. (a) CWT of the spindle current signal; (b) DWT of feed current signal, resolution $j=2$.

result of DWT analysis of feed current at resolution $j=2$, respectively. Tool breakage is indicated by above processing.

To detect the tool breakage efficiently in drilling process monitoring way showed fit into the kinds of cutting conditions. Figs. 4, 6 and 8 show the spindle current signal and feed current signal under the different cutting condition, respectively. Figs. 5, 7 and 9 are the results of above signal processing,respectively. Clearly small differences between the normal and tool breakage can be observed above processed signals. Using signal analysis, it was confirmed that proposed methodlogy can reliably detect tool breakage (Figs. 4-9).
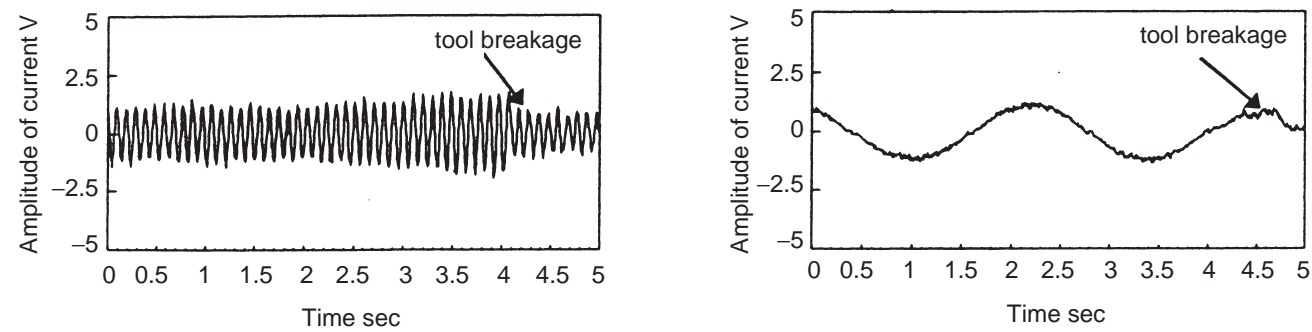

Fig. 6. Live tool breakage current signals, cutting speed: $300 \mathrm{r} / \mathrm{min}$, feed: $30 \mathrm{~mm} / \mathrm{min}$, drill diameter: $1 \mathrm{~mm}$. (a) Spindle current signal; (b) feed current signal. 

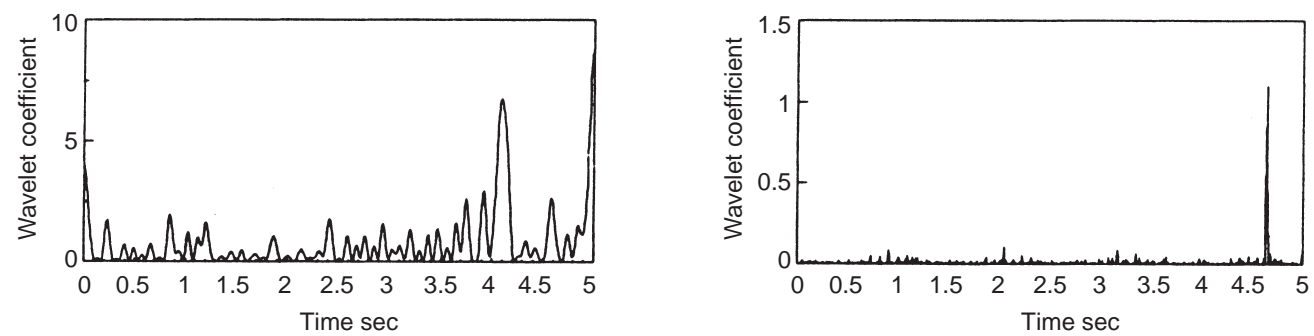

Fig. 7. WT of current signal, cutting speed: $300 \mathrm{r} / \mathrm{min}$, feed: $30 \mathrm{~mm} / \mathrm{min}$, drill diameter: $1 \mathrm{~mm}$. (a) CWT of the spindle current signal; (b) DWT of feed current signal, resolution $j=2$.
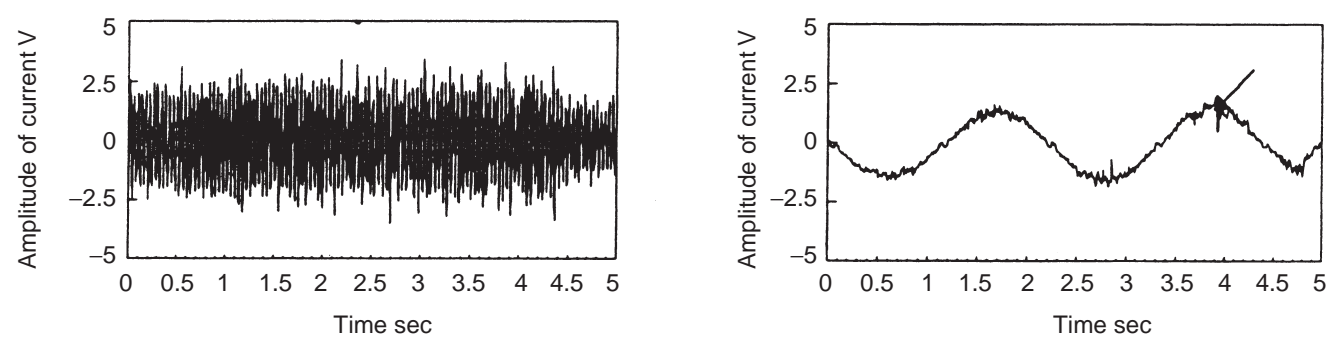

Fig. 8. Live tool breakage current signals, cutting speed: $450 \mathrm{r} / \mathrm{min}$, feed: $45 \mathrm{~mm} / \mathrm{min}$, drill diameter: $4.5 \mathrm{~mm}$. (a) Spindle current signal; (b) feed current signal.
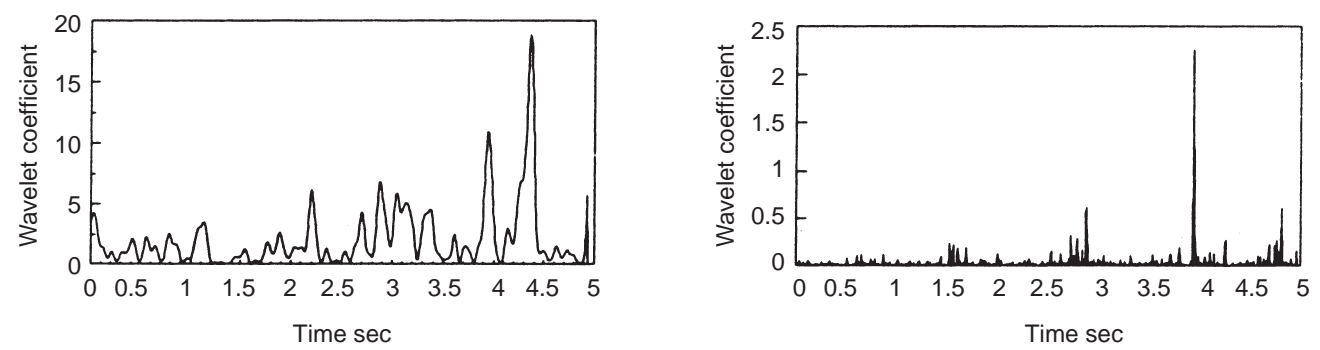

Fig. 9. WT of current signal, cutting speed: $450 \mathrm{r} / \mathrm{min}$, feed: $45 \mathrm{~mm} / \mathrm{min}$, drill diameter: $4.5 \mathrm{~mm}$. (a) CWT of the spindle current signal; (b) DWT of feed current signal, resolution $j=2$.

\section{Conclusions}

In this paper the AC servo motor current was indirectly measured and the WT was used to detect the tool breakage is the drilling process for small diameter drill. The experimental results conducted show that DWT can clearly detect the small diameter drill tool breakage. The developed current sensoring system has been applied to the on-line detection of tool breakage in drilling process. The experimental set-up can be attached to commercial MCs without changes of the machine. 


\section{References}

[1] D.A. Dornfeld, Neural network sensor fusion for tool condition monitoring, Annals of the CIRP 39 (1) (1990) 101-1051.

[2] B. Byrne, D.A. Dornfeld et al., Tool condition monitoring (TCM) - the status of research and industrial application, Annals of the CIRP 44 (2) (1995) 541-567.

[3] L. Dan, J. Mathew, Tool wear and failure monitoring techniques for turning - a review, Int. J. Mach. Tools Manufact. 30 (4) (1990) 579-598.

[4] K.F. Marti, J.A. Brandon, B.I. Grosvenor, A. Dwen, A comparison of in-process tool wear measurement methods in turning. Proc. 26th. Int. Machine Tool Design and Research Conf., 1986, pp. 289-296.

[5] A. Novak, G. Ossbahr, Reliability of the cutting force monitoring in FMS-installations. Proc. 26th Int. Machine Tool Design and Research Conf., 1986, pp. 325-329.

[6] Daubechies, The wavelet transform, time-frequency localization and signal analysis. IEEE Transactions on Information Theory 36(5) (1990) 961-1005.

[7] Daubechies, Orthogonal bases of compactly supported wavelets. Communications on Pure and Applied Mathematics XLI (1988) 909-996.

[8] I. Tansel, Detection of tool failure in end milling with wavelet transformations and neural networks (WT-NN), Int. J. Mach. Tools Manufact. 35 (4) (1995) 1137-1147.

[9] I. Tansel, C. Mekdeci, O. Rodriguez, B. Uragun, Monitoring drill conditions with wavelet based encoding and neural network, Int. J. Mach. Tools Manufact. 33 (4) (1995) 559-575.

[10] N. Kasashima, On-line failure detection in face milling using discrete wavelet transform, Annals of the CIRP 44 (1) (1995) 483-487.

[11] T. Nagao, Y. Hatamura, M. Mitsuishi, In-process prediction and prevention of the breakage of small diameter drills-based on theoetical analysis, Annals of the CIRP 43 (1) (1994) 85-88.

[12] O. Rioul, P. Duhamel, Fast algorithms for discrete and continuous wavelet transforms, IEEE Trans. on Information Theory 38 (2) (1992) 569-585.

[13] G. Evangelisto, Orthogonal wavelet transforms and filter banks. Presented at Proc. 23rd Asilomar Conf. IEEE, 1989. 